\title{
Time Domain Propagation Characteristics with Causal Channel Model for Terahertz Band
}

\author{
Zhaona $\mathrm{Wu}^{*}$, Hiroto Ebisawa**, Kenta Umebayashi***, Janne Lehtomäki ${ }^{\dagger}$, Nizar Zorba ${ }^{\ddagger}$ \\ * Tokyo University of Agriculture and Technology, zhaonawu@st.go.tuat.ac.jp \\ ** Tokyo University of Agriculture and Technology, s205631v@st.go.tuat.ac.jp \\ *** Tokyo University of Agriculture and Technology, ume_k@cc.tuat.ac.jp \\ $\dagger$ Centre for Wireless Communications, University of Oulu, janne.lehtomaki@ee.oulu.fi \\ $\ddagger$ Qatar University, nizarz@qu.edu.qa
}

\begin{abstract}
In recent years, as more devices are connected to wireless communication systems, the demand for spectrum has increased. As conventional spectrum resources are limited, the THz band becomes an interesting option of more spectrum for wireless communication. However, the channel in $\mathrm{THz}$ band has different characteristics compared to the channels in typical frequency bands, and therefore, it is necessary to perform more research to understand the $\mathrm{THz}$ channel propagation. In this paper, we focus on the time domain $\mathrm{THz}$ channel model under line of sight $(\mathrm{LOS})$ propagation conditions and investigate the channel propagation characteristics in time domain. Firstly, in the full frequency band (FFB) scenario, the time domain impulse responses, which correspond to the time domain $\mathrm{THz}$ channel model, are presented for different distances. In the impulse responses, there are significantly delayed paths due to the molecular absorption which causes significant frequency selectivity. Secondly, we extend the model to the limited frequency band (LFB) scenario by applying the root raised cosine filters. The results indicate that the richness of the delayed paths in the impulse response depends on the selected frequency band. In addition, the results indicate that the time delay and total energy strongly depend on the distance whereas the delay spread varies as a function of frequency.

Index Terms-Average delay, delay spread, impulse response, THz, total energy.
\end{abstract}

\section{INTRODUCTION}

In the wireless communication field, spectrum scarcity is an urgent issue since the demand for spectrum resources is increasing year by year, but the spectrum is a finite resource and the most of accessible spectrum has been allocated to the existing wireless systems/services [1]. Several approaches to solve the spectrum scarcity problem have been proposed. One of the promising approaches is the usage of higher frequency bands such as millimeter-wave (mmWave) band and terahertz band (THz band). A significant benefit of wireless communication in $\mathrm{THz}$ band is the huge amounts of bandwidth, capable of providing high data rates and/or serving a large number of users such as eMBB (enhanced Mobile Broadband) and mMTC (massive Machine Type Communication). Wireless communications in $\mathrm{THz}$ has been expected to be used in Beyond 5G and 6G Systems [2].

For system deployment at $\mathrm{THz}$ band, characteristics of propagation in $\mathrm{THz}$ have to be investigated and understood.
Traditional wireless communication systems operate at significantly lower frequencies compared to $\mathrm{THz}$, such as ultra-high frequency band (UHF: $300 \mathrm{MHz}-3 \mathrm{GHz}$ ), where extensive investigations on channel modeling have been made [3].

The propagation in $\mathrm{THz}$ band has two important aspects [4]. The first one is that path loss is significant due to the high frequency, and the second one is that molecular absorption leads to significant frequency selectivity even in a LoS case, which contributes to the significantly low gain at certain frequencies.

To understand the propagation in $\mathrm{THz}$, and to provide appropriate channel models for the $\mathrm{THz}$ wireless communications, previous works have been carried out. In [5], a channel model incorporates the propagation aspects such as the LoS, reflected, scattered, and diffracted paths by using the ray tracing. However, the channel model may not fit the wide-band scenario where molecular absorption leads to frequency selectivity. In [6], non-stationary channel models for the mmWave band were proposed. In [7], a stochastic channel model is proposed for $300 \mathrm{GHz}$ where the frequency scattering for indoor scenarios is considered.

In [5], the channel models are proposed by adding a linear phase component into the transmittance. Specifically, the phase component of the frequency response is set based on linear phase criterion, and after that, the channel impulse response is obtained by using inverse Fourier Transform (IFT). However, these channel models don't satisfy causality. Causality requires that there is no output from the channel before it is physically possible (for example taking into account the propagation distance under the LoS conditions). In [8], the phase component in the channel model is obtained based on minimum phase, thus satisfying causality. The investigations in [8] are limited to the short-range $(1-100 \mathrm{~cm})$ communication and some important channel characteristics, such as average delay, delay spread, and total energy in time domain have not been shown.

In this paper, we have investigated the time domain channel model for longer distance cases for different $\mathrm{THz}$ frequency bands. In addition, The $\mathrm{THz}$ channel model propagation characteristics (average delay, delay spread, and total energy) in time domain are thoroughly presented and analyzed. 
The rest of this paper is organized as follows. Section II describes the system model followed by Section III with the channel model for the FFB scenario. The channel model for the LFB scenario is tackled in Section IV, while the channel propagation characteristics are discussed in Section V. Finally, the conclusions are presented in Section VI.

\section{SySTEM MODEL}

A LoS case in $\mathrm{THz}$ band is assumed. In free space, molecular absorption and spreading loss are the main loss mechanisms. Antenna gain is assumed to be $0 \mathrm{~dB}$ without loss of generality. The considered propagation models are shown in Figs. 1 and 2 in which there are the transmitter, receiver, and the channel between them. The first model in Fig. 1 is the FFB, whose frequency band is full terahertz band $(0.1$ to $10 \mathrm{THz}$ ), and it would show the inherent characteristics of the $\mathrm{THz}$ channel propagation. In the second model which corresponds to the LFB in Fig. 2, there are band pass filters (BPFs) at the transmitter and receiver, respectively. In this case, the total channel includes the actual channel and the band pass filters. Typical wireless communication is based on the LFB and it would show the inherent effect of the channel on the wireless communication.

The common parameters used in all results are as follows: Pressure $p=1010 \mathrm{hPa}, \mathrm{RH}=69.6 \%$, and temperature $T=$ $298.55 \mathrm{~K}$

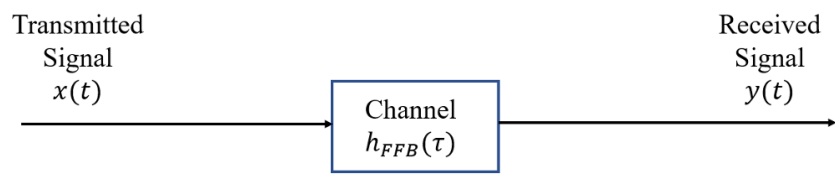

Fig. 1. Propagation model in FFB secenario.

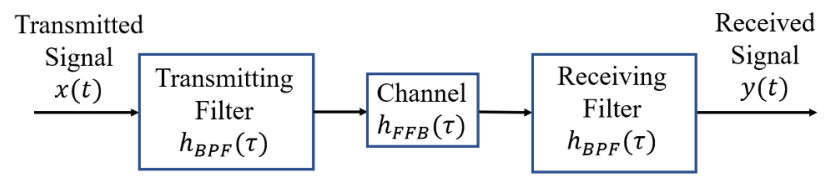

Fig. 2. Propagation model in LFB secenario.

\section{Channel Model in Full FreQuency BAnd (FFB) SCENARIO}

\section{A. Transmittance}

When the signal goes through the channel, the electromagnetic wave suffers attenuation due to the molecular absorption. Then the radiative transfer theory [9] and the information provided by the HITRAN database [10] are used to compute the attenuation. The parameter transmittance $\left|H_{F F B}(f, r)\right|^{2}$ is defined as [8]:

$$
\left|H_{F F B}(f, r)\right|^{2}=\frac{P_{r}(f, r)}{P_{t}(f, r=0)}
$$

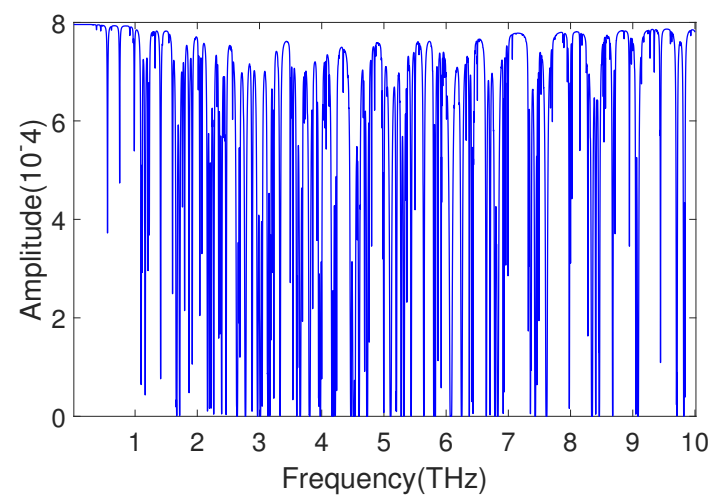

Fig. 3. Transmittance $\left|H_{F F B}(f, r)\right|^{2}$ as a function of frequency, distance $r=10 \mathrm{~cm}$.

where $f$ is the frequency, $r$ is the propagation distance between the transmitter and the receiver. $P_{t}(f, r=0)$ is the transmitted signal power and $P_{r}(f, r)$ is the received signal power. $H_{F F B}(f, r)$ is the frequency response.

When molecular absorption loss and spreading loss are considered, the transmittance (1) can be calculated as [11]:

$$
\left|H_{F F B}(f, r)\right|^{2}=\left[A_{\text {abs }}(f, r) \times A_{\text {spread }}(r)\right]^{-1}
$$

where $A_{a b s}(f, r)$ is the molecular absorption loss and $A_{\text {spread }}(r)$ is the spreading loss.

The molecular absorption loss $A_{a b s}(f, r)$ can be found with [12]:

$$
A_{a b s}(f, r)=A_{l}(f, r) \times A_{c}(f, r)
$$

with $A_{l}(f, r)$ and $A_{c}(f, r)$ given as

$$
A_{l}(f, r)=\exp \left(\sum_{m} k_{l}^{\mathrm{m}}(f) r\right)
$$

and

$$
A_{c}(f, r)=\exp \left(\sum_{n} k_{c}^{\mathrm{n}}(f) r\right)
$$

where $A_{l}(f, r)$ is the line absorption loss, $A_{c}(f, r)$ is the continuum absorption loss, $m$ and $n$ are indices for molecular species and the source of the continuum absorption, respectively and $k_{l}$ and $k_{c}$ are the line absorption coefficient and the continuum absorption coefficient, respectively. In the calculation of the line absorption and continuum absorption coefficients, the am model [12] and the famous HITRAN catalog [10] are used.

For an ideal isotropic transmitter, the spreading loss in the LoS path is

$$
A_{\text {spread }}(r)=4 \pi r^{2}
$$

Fig. 3 shows that the transmittance depends on the frequency. That means electromagnetic wave suffers from frequency-selective channel due to the molecular absorption. 


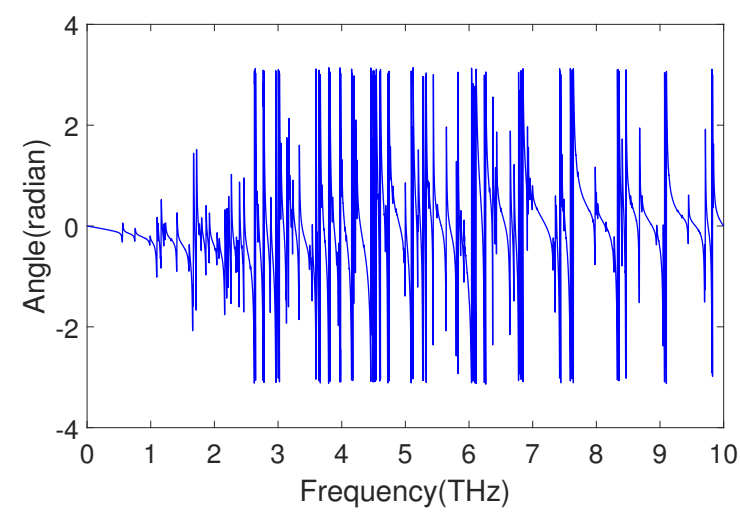

Fig. 4. Phase response $\psi(f, r)$ as a function of frequency, distance $r=10$ $\mathrm{cm}$.

\section{B. Minimum Phase}

From Eq. (2), the amplitude response of the frequency response is included in the transmittance, but not the phase response. For the phase component, we use the minimum phase to make a causal channel model. In [8], this approach has been confirmed experimentally, but the previous linear phase response has been shown to give erroneous results.

Impulse response with causality of the LoS path satisfies:

$$
h_{F F B}(\tau)= \begin{cases}h_{F F B}(\tau, r), & \tau \geq \tau_{l} \\ 0, & \tau<\tau_{l}\end{cases}
$$

where $\tau_{l}$ is the propagation delay of the LoS path and given by $\tau_{l}=r / c$.

The frequency response of $h_{F F B}(\tau, r)$ is given by

$$
\begin{aligned}
H_{F F B}(f, r) & =\int_{\tau_{l}}^{\infty} h_{F F B}(\tau, r) e^{-\mathrm{j} 2 \pi \mathrm{f} \tau} d \tau \\
& =e^{-j 2 \pi f \tau_{l}} \int_{0}^{\infty} h_{F F B}\left(\tau+\tau_{l}, r\right) e^{-j 2 \pi f \tau} d \tau
\end{aligned}
$$

where

$$
H_{F F B}^{\prime}(f, r)=\int_{0}^{\infty} h_{F F B}\left(\tau+\tau_{l}, r\right) e^{-j 2 \pi f \tau} d \tau
$$

is defined as causal frequency response in the channel model. Then

$$
H_{F F B}(f, r)=e^{-j 2 \pi f \tau_{l}} H_{F F B}^{\prime}(f, r) .
$$

We define the amplitude and phase of $H_{F F B}^{\prime}(f, r)$

$$
\begin{gathered}
\left|H_{F F B}^{\prime}(f, r)\right|=\exp (-\beta(f, r)) \\
\arg \left(H_{F F B}^{\prime}(f, r)\right)=\psi(f, r) .
\end{gathered}
$$

Then

$$
H_{F F B}^{\prime}(f, r)=\exp [-\beta(f, r)+j \psi(f, r)] .
$$

$\beta(f, r)$ and $\psi(f, r)$ are Hilbert transform pairs [12] [13] to make the channel causal. Therefore

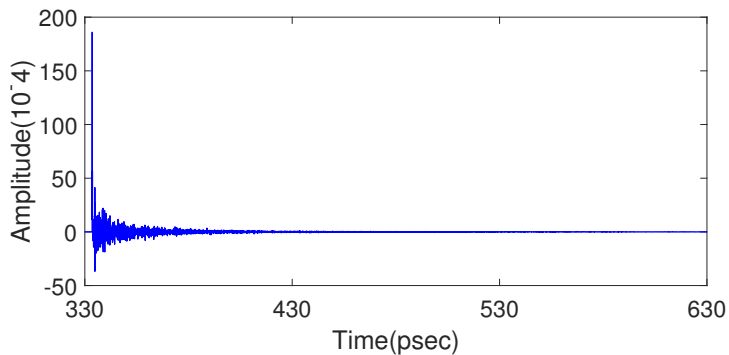

(a)

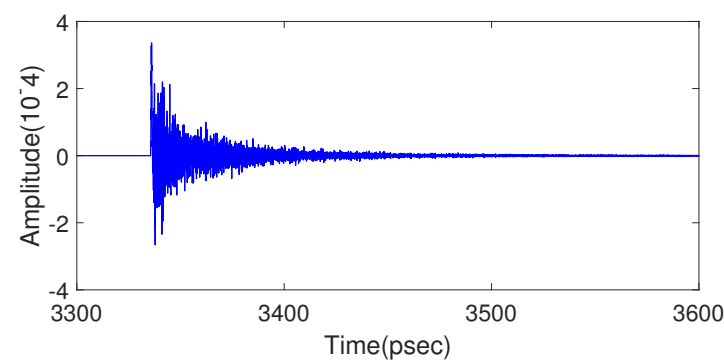

(b)

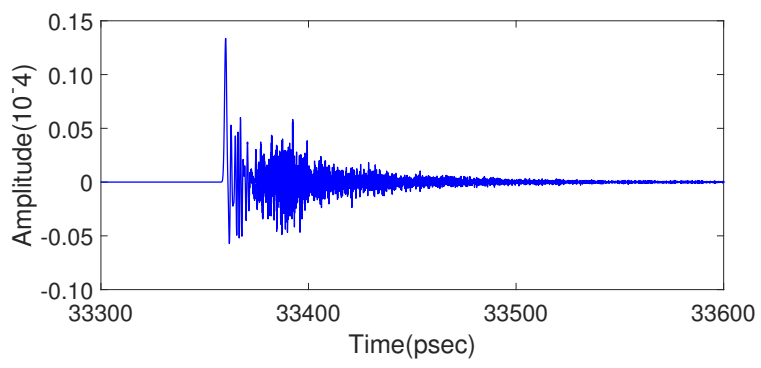

(c)

Fig. 5. Impulse response $h_{F F B}(\tau)$ as a function of $\tau$. (a)-(c) Distance $r=10 \mathrm{~cm}, r=1 \mathrm{~m}, r=10 \mathrm{~m}$.

$$
\psi(f, r)=\frac{1}{\pi} P V \int_{-\infty}^{\infty} \frac{\beta\left(f^{\prime}, r\right)}{f-f^{\prime}} d f^{\prime}
$$

where $P V$ represents Cauchy principal value. The phase response $\psi(f, r)$ in Eq. (14) is defined as minimum phase which is shown in Fig. 4.

\section{Impulse Response in the FFB scenario}

According to (10)-(14), we obtain the frequency response (including amplitude and phase responses) of the causal channel model.

Inverse Fourier Transform can be applied to get the impulse response. Finally the causal impulse response is as follows:

$$
h_{F F B}(\tau)=\mathcal{F}^{-1}\left[H_{F F B}(f, r)\right] .
$$

\section{Numerical Results}

In this section, we show some simulations of impulse response at different distances in the FFB scenario. We can see the characteristic of impulse response in terms of distance.

From Fig. 5, we can confirm that there are significantly delayed paths due to the molecular absorption. This aspect is 
very unique in $\mathrm{THz}$ propagation. In addition, the channel gain decays quickly as the distance increases. Specifically, distance increasing from $10 \mathrm{~cm}$ to $1 \mathrm{~m}$, the channel gain decays more significantly than the case of distance increasing from $1 \mathrm{~m}$ and $10 \mathrm{~m}$.

\section{Channel Model in Limited Frequency Band (LFB) SCENARIO}

\section{A. Band Pass Filter}

In the real case, the wireless communication has to be on the LFB by a BPF. In the LFB scenario, there are two root raised cosine filters as BPFs at the transmitter and receiver sides. The frequency response of BPF is given [14]

$H_{B P F}(f)= \begin{cases}1, & \left|f-f_{c}\right|<2 W_{0}-W \\ \cos \left(\frac{\pi}{4} \frac{\left|f-f_{c}\right|+W-2 W_{0}}{W-W_{0}}\right), & 2 W_{0}-W<\left|f-f_{c}\right|<W \\ 0, & \left|f-f_{c}\right|>W\end{cases}$

where $W=B / 2, B$ is the bandwidth of BPF. $W_{0}=$ $W /(a+1)$ represents the minimum Nyquist bandwidth for the rectangular spectrum and the half-amplitude point for the raised-cosine spectrum. $f_{c}$ is the central frequency. The rolloff factor $a$ is set to $a=1$.

\section{B. Impulse Response in the LFB Scenario}

According to the Eq. (10) and (16), we can get the channel frequency response in the LFB scenario which is given by

$$
H_{L F B}(f, r)=H_{B P F}(f) H_{F F B}(f, r) H_{B P F}(f) .
$$

Then using Inverse Fourier Transform, we get the channel impulse response in the LFB scenario which is given by

$$
h_{L F B}(\tau)=\mathcal{F}^{-1}\left[H_{L F B}(f, r)\right] .
$$

\section{Numerical results}

Impulse responses based on LFB in (18) in different center frequencies at different distances are shown as follows. The center frequencies are set to $5.15 \mathrm{THz}$ and $7.15 \mathrm{THz}$, respectively and, the assumed bandwidth is $0.3 \mathrm{THz}$.

Significant frequency selectivity at $5.15 \mathrm{THz}$ leads to a richness of the delayed paths in the impulse responses as we can confirm from Figs. 6-8. In addition, different distances lead to different types of impulse responses. This aspect would affect wireless communication performance since this would cause inter-symbol-interference, especially for pulsebased communication. Therefore, it is necessary to understand the channel characteristics and provide an appropriate model of the delayed paths in terms of frequency and distance.

\section{Channel propagation Characteristics in time DOMAIN}

The important characteristics of the time domain channel can be evaluated by the average delay, delay spread, and total energy as a function of frequency in terms of different distances. In this investigation, the bandwidth is set to $0.5 \mathrm{THz}$ and we consider the LFB scenario.

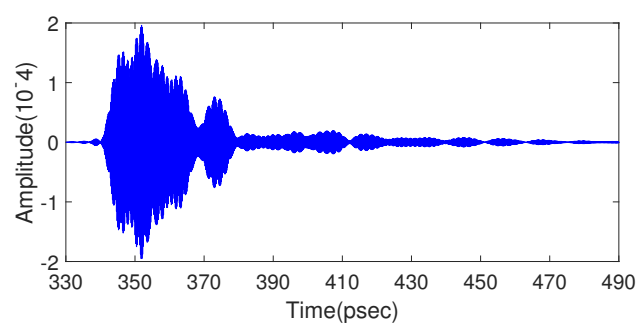

(a)

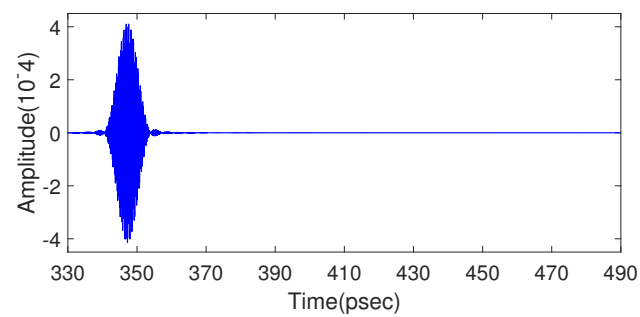

(b)

Fig. 6. Impulse response $h_{L F B}(\tau)$ as a function of $\tau$. (a) $r=10 \mathrm{~cm}$, $f_{c}=5.15 \mathrm{THz}, B=0.3 \mathrm{THz}$, (b) $r=10 \mathrm{~cm}, f_{c}=7.15 \mathrm{THz}, B=0.3$ THz.

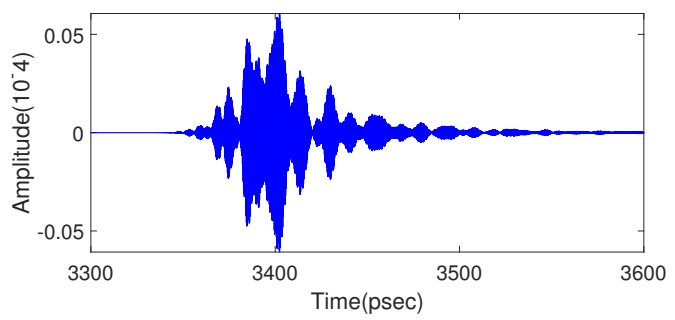

(a)

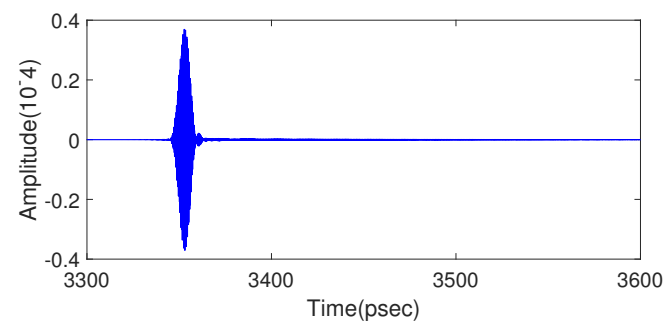

(b)

Fig. 7. Impulse response $h_{L F B}(\tau)$ as a function of $\tau$. (a) $r=1 \mathrm{~m}, f_{c}=$ $5.15 \mathrm{THz}, B=0.3 \mathrm{THz}$, (b) $r=1 \mathrm{~m}, f_{c}=7.15 \mathrm{THz}, B=0.3 \mathrm{THz}$.

\section{A. Average Delay}

Average delay is defined by [15]

$$
\tau_{m}=\frac{\int_{0}^{\infty} \tau|h(\tau)|^{2} d \tau}{\int_{0}^{\infty}|h(\tau)|^{2} d \tau}
$$

and this indicates the mean of delay weighted by the channel gain. Specifically, the average delay is determined by not only the propagation delay $\tau_{l}=r / c$, but also multiple delayed paths in the impulse responses.

From Fig. 9, the results show that the average delay is mainly determined by $\tau_{l}$. In addition, we can also confirm 


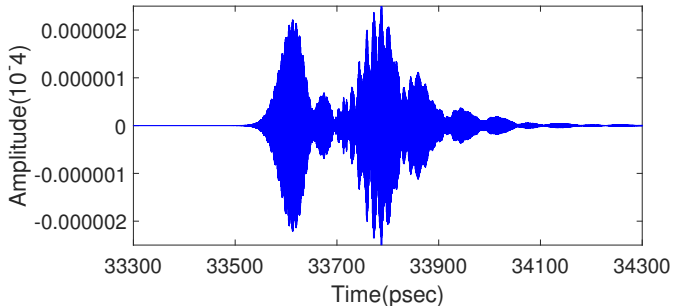

(a)

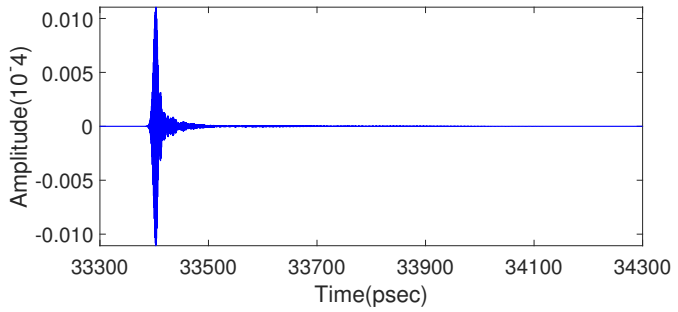

(b)

Fig. 8. Impulse response $h_{L F B}(\tau)$ as a function of $\tau$. (a) $r=10 \mathrm{~m}$, $f_{c}=5.15 \mathrm{THz}, B=0.3 \mathrm{THz}$, (b) $r=10 \mathrm{~m}, f_{c}=7.15 \mathrm{THz}, B=0.3$ THz.

the fluctuation of average delay for a different frequency. This may depend on the richness of the delayed paths. For example, in case of $5.15 \mathrm{THz}$ and $r=10 \mathrm{~cm}, \tau_{m}=3.5 \cdot 10^{-10} \mathrm{sec}$, but in case of $7.15 \mathrm{THz}$ and $r=10 \mathrm{~cm}, \tau_{m}=3.4 \cdot 10^{-10} \mathrm{sec}$.

\section{B. Delay Spread}

The delay spread is defined in literature by [15]

$$
\tau_{s}=\sqrt{\frac{\int_{0}^{\infty}\left(\tau-\tau_{m}\right)^{2}|h(\tau)|^{2} d \tau}{\int_{0}^{\infty}|h(\tau)|^{2} d \tau}}
$$

and Fig. 10 shows the delay spread within our scenario as a function of the center frequency. For an appropriate design of wireless communications in $\mathrm{THz}$, this information is important since this is related to inter-symbol-interference. The result in Fig. 10 indicates that longer distance leads to more variability of $\tau_{s}$ in the frequency domain. For the longdistance, such as $r=10 \mathrm{~m}$, the channel gain at $\tau_{l}$ is attenuated significantly compared to the short distance case. Therefore, the significance of the channel gain at $\tau_{l}$ may not be dominant in the case of long distance which leads to the variability of $\tau_{s}$.

\section{Total Energy}

The total energy is defined as

$$
p=\int_{0}^{\infty}|h(\tau)|^{2} d \tau
$$

This depends on spreading loss and loss due to the molecular absorption at the frequency. The total energy as a function of frequency is shown in Fig. 11. As the distance increases, the total energy decreases significantly. The fluctuation of total energy is caused by molecular absorption and this fact has to be considered for the design of wireless communication.

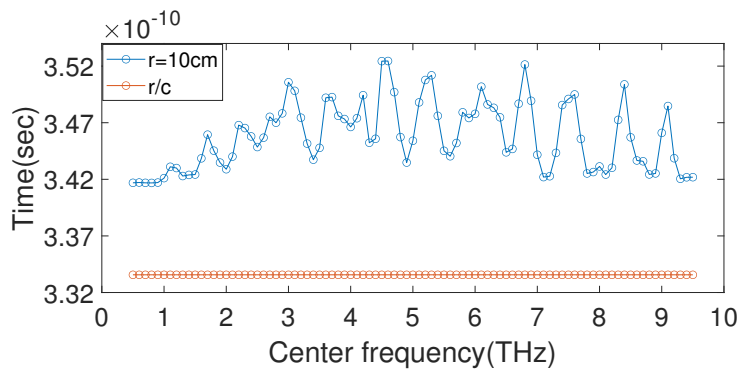

(a)

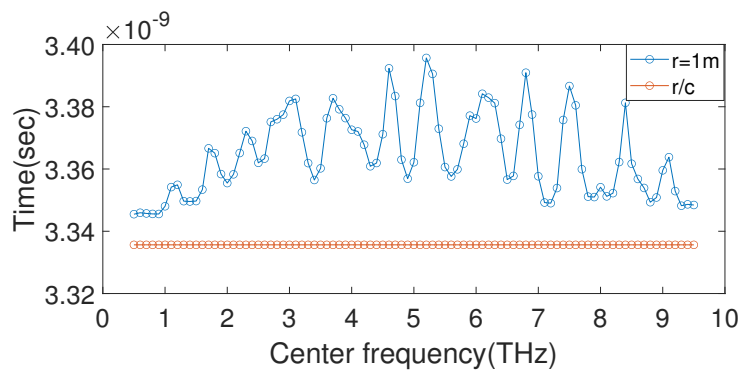

(b)

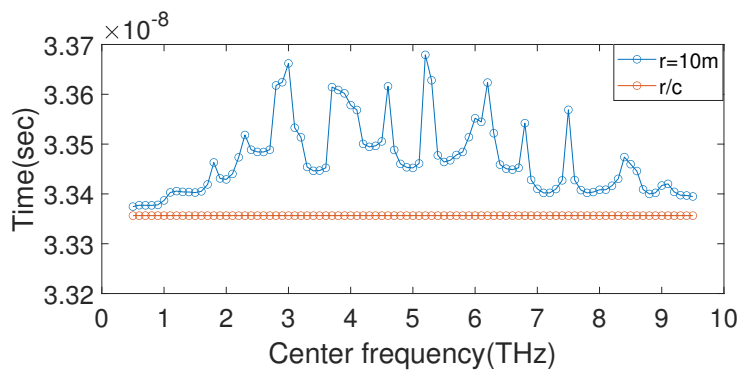

(c)

Fig. 9. Comparison between average delay and the arrival time of first tap $(r / c)$. (a)-(c) Distance $r=10 \mathrm{~cm}, r=1 \mathrm{~m}, r=10 \mathrm{~m}$.

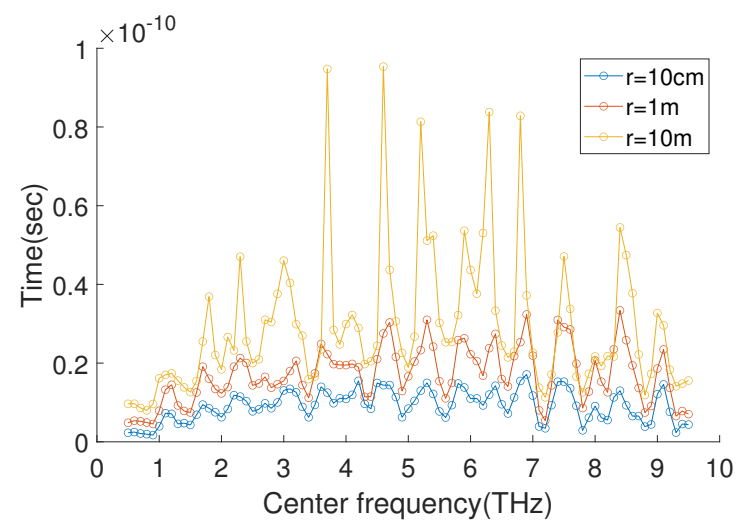

Fig. 10. Delay spread.

\section{CONCLUSIONS}

In this paper, we have investigated the impulse responses as the time domain channel model for $\mathrm{THz}$ wireless communications. Due to the molecular absorption loss, there is significant frequency selectivity, and this leads to delayed paths in the im- 


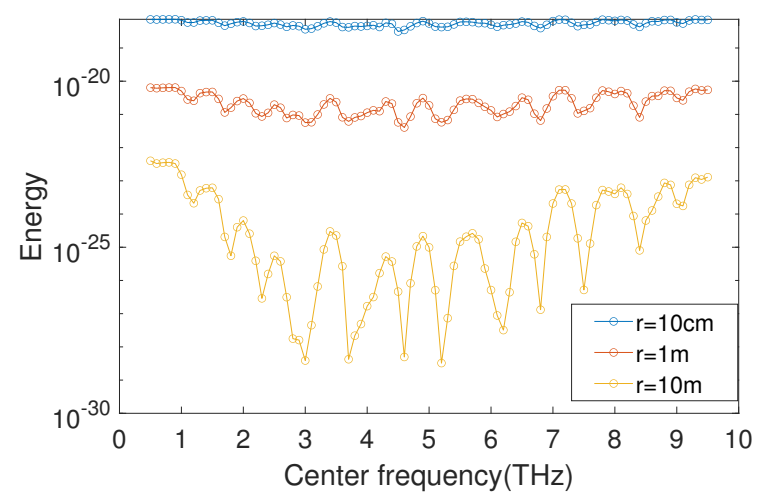

Fig. 11. Total energy.

pulse responses. We have confirmed that the impulse responses in the limited frequency band scenario strongly depend on the frequency band. In addition, this aspect strongly affects the delay spread. On the other hand, in our investigations, average delay and total energy are mainly affected by propagation delay $\tau_{l}$ while the molecular absorption also provides some influences to average delay and total energy.

\section{ACKNOWLEDGEMENT}

This work was partially funded by project Marubeni - Qatar University number M-QJRC-2020-4. The statements made herein are solely the responsibility of the authors. The work of J. Lehtomäki was supported by the Academy of Finland 6Genesis Flagship (grant no. 318927).

\section{REFERENCES}

[1] M. Song, C. Xin, Y. Zhao, and X. Cheng, "Dynamic spectrum access: from cognitive radio to network radio,' IEEE Wireless Communications, vol. 19, no. 1, pp. 23-29, 2012.

[2] W. Saad, M. Bennis, and M. Chen, "A vision of 6G wireless systems: Applications, trends, technologies, and open research problems," IEEE network, vol. 34, no. 3, pp. 134-142, 2019.

[3] ITU-R, "Multipath propagation and parameterization of its characteristics," 1999.

[4] B. Sklar, "Rayleigh fading channels in mobile digital communication systems. i. characterization," IEEE Communications magazine, vol. 35 , no. 7, pp. 90-100, 1997.

[5] C. Han, A. O. Bicen, and I. F. Akyildiz, "Multi-ray channel modeling and wideband characterization for wireless communications in the terahertz band," IEEE Transactions on Wireless Communications, vol. 14, no. 5, pp. 2402-2412, 2014.

[6] Y. Liu, C.-X. Wang, J. Huang, J. Sun, and W. Zhang, "Novel 3-D nonstationary mmwave massive MIMO channel models for 5G highspeed train wireless communications," IEEE Transactions on Vehicular Technology, vol. 68, no. 3, pp. 2077-2086, 2018.

[7] S. Priebe and T. Kurner, "Stochastic modeling of $\mathrm{THz}$ indoor radio channels," IEEE Transactions on Wireless Communications, vol. 12, no. 9, pp. 4445-4455, 2013.

[8] K. Tsujimura, K. Umebayashi, J. Kokkoniemi, J. Lehtomäki, and Y. Suzuki, "A causal channel model for the terahertz band," IEEE Transactions on Terahertz Science and Technology, vol. 8, no. 1, pp. 52-62, 2017.

[9] R. M. Goody and Y. L. Yung, Atmospheric radiation: theoretical basis. Oxford university press, 1995.

[10] L. S. Rothman, I. E. Gordon, A. Barbe, D. C. Benner, P. F. Bernath, M. Birk, V. Boudon, L. R. Brown, A. Campargue, J.-P. Champion et al., "The HITRAN 2008 molecular spectroscopic database," Journal of Quantitative Spectroscopy and Radiative Transfer, vol. 110, no. 9-10, pp. 533-572, 2009.
[11] J. M. Jornet and I. F. Akyildiz, "Channel modeling and capacity analysis for electromagnetic wireless nanonetworks in the terahertz band," IEEE Transactions on Wireless Communications, vol. 10, no. 10, pp. 32113221, 2011.

[12] S. Paine, "The am atmospheric model," Zenodo, 2012.

[13] H. Kuzmany, Solid-state spectroscopy: an introduction. Springer Science \& Business Media, 2009.

[14] B. Sklar et al., Digital communications: fundamentals and applications, 2001.

[15] J. D. Parsons, The mobile radio propagation channel. Wiley, 2000. 